\title{
RHIC COMMISSIONING AND FIRST RESULTS
}

\author{
S. Ozaki, Brookhaven National Laboratory, Upton, NY 11973, USA
}

\section{Abstract}

The Relativistic Heavy Ion Collider (RHIC) at Brookhaven National Laboratory is the U.S. Department of Energy's flagship research facility for the nuclear physics program. The construction of the collider and a complementary set of detectors were completed as scheduled during 1999. Following the initial verification of functionalities of the collider in the same year, collisions of $\mathrm{Au}$ ions were achieved during the subsequent commissioning run in the year 2000, first at the collision energy of $56 \mathrm{GeV}$ per nucleon on June 12 and later at 132 $\mathrm{GeV}$ per nucleon. All four detectors, BRAHMS, PHENIX, PHOBOS, and STAR, were also commissioned and collected significant amounts of data during the fourweek run period. This report gives an overview of the RHIC facility, its commissioning activities, and the first physics results at the new frontier of nuclear matter research that is opened by RHIC.

\section{INTRODUCTION}

It is a great honor to have been invited to give the opening talk of PAC2001 on RHIC and its First Physics Results. On behalf of all of those who have engaged in the design, construction, commissioning of the RHIC collider and detectors, and worked for the first physics results from BNL as well as elsewhere, I thank the program committee of this conference for their recognition of the achievements made by our team.

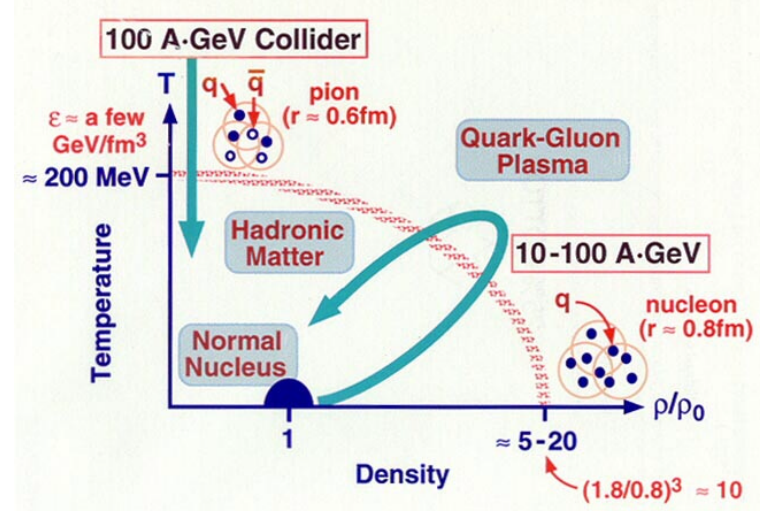

Figure 1: Phase Diagram of Nuclear Matter.

RHIC is a research facility that was built to satisfy the quest of mankind to understand how this universe was born. Fig. 1 shows the phase diagram of nuclear matter as the function of baryon density and temperature, with a contour showing the predicted phase transition from the hadronic matter to the quark-gluon plasma (QGP). Toward the far right on the horizontal axis one finds an extremely high baryon density state, such as in the neutron star where one may find the quark-gluon plasma phase. Going up along the temperature axis, the figure indicates the phase transitions into the QGP domain at a sufficiently high temperature. In fact, it is expected that the collisions of $\mathrm{Au}$ ions at the beam energy of 100 $\mathrm{GeV} /$ nucleon at RHIC will result in the state of matter at a temperature of about $10^{12} \mathrm{~K}$. The primary objective of RHIC, therefore, is to investigate the phase transition and to study the formation and property of QGP. With RHIC, nuclear physics is entering into the high-energy domain.

\section{THE RHIC FACILITY}

The idea to build RHIC dates back to 1983, when it was conceived as part of the long-range plan of US nuclear science. Construction of the facility began in 1991, was completed in 1999, and its physics program began in 2000, 17 years after its conception. Design parameters of the collider are given in Table 1.

Table 1: Performance Specifications of RHIC

\begin{tabular}{|l|l|l|}
\hline & for $\mathrm{Au}-\mathrm{Au}$ & for $\mathrm{p}-\mathrm{p}$ \\
\hline Beam Energy & $100 \rightarrow 30 \mathrm{GeV} / \mathrm{u}$ & $250 \rightarrow 30 \mathrm{GeV}$ \\
\hline Luminosity & $2 \times 10^{26} \mathrm{~cm}^{-2} \mathrm{sec}^{-1}$ & $1.4 \times 10^{31} \mathrm{~cm}^{-2} \mathrm{sec}^{-1}$ \\
\hline Number of Bunches/Ring & $60(\rightarrow 120)$ & $60(\rightarrow 120)$ \\
\hline Luminosity Lifetime & $\sim 10 \mathrm{hrs}$ & $>10 \mathrm{hrs}$ \\
\hline$\beta^{*}$ at Collision Points & $10 \mathrm{~m} \rightarrow 2 \mathrm{~m}(1 \mathrm{~m} ?)$ & $10 \mathrm{~m} \rightarrow 2 \mathrm{~m}(1 \mathrm{~m} ?)$ \\
\hline
\end{tabular}

The collider consists of two quasi-circular concentric accelerator/storage rings on a common horizontal plane, one ("Blue Ring") for clockwise and the other ("Yellow Ring") for counterclockwise beams. Rings are oriented to intersect with one another at six locations along their 3.8 $\mathrm{km}$ circumference. Each ring consists of 6 arc sections (each $\sim 356 \mathrm{~m}$ long) and 6 insertion sections (each $\sim 277 \mathrm{~m}$ long) with a collision point at their center. In the arc sections, the counter rotating beams are separated by 90 $\mathrm{cm}$ horizontally. A pair of dipole magnets, located at $\sim 10$ $\mathrm{m}$ and at $\sim 23 \mathrm{~m}$ from the collision point, steer beams to a co-linear path for head-on collisions. Three quadrupole magnets, located outside the steering dipoles form the final focus triplet for high luminosity collisions. Superconducting magnets are used exclusively for both storage rings. Altogether, 1,740 superconducting magnets are used for the RHIC collider, of which 1200 units were manufactured by industry.

The RHIC acceleration scenario for $\mathrm{Au}$ ion beams is shown in Fig. 2. Three accelerators in the injector string will successively boost the energy of ions, and strip electrons from atoms. Fully stripped gold ions are injected into the RHIC rings.

Acceleration and storage of beam bunches at RHIC require two rf systems; i.e., one operating at $28 \mathrm{MHz}$ to capture the AGS bunches and accelerate to the top energy, and the other operating at $197 \mathrm{MHz}$ to provide short collision diamonds $(\sigma \sim 25 \mathrm{~cm})$ for a more reasonable 
detector design. The synchrotron phase transition of the

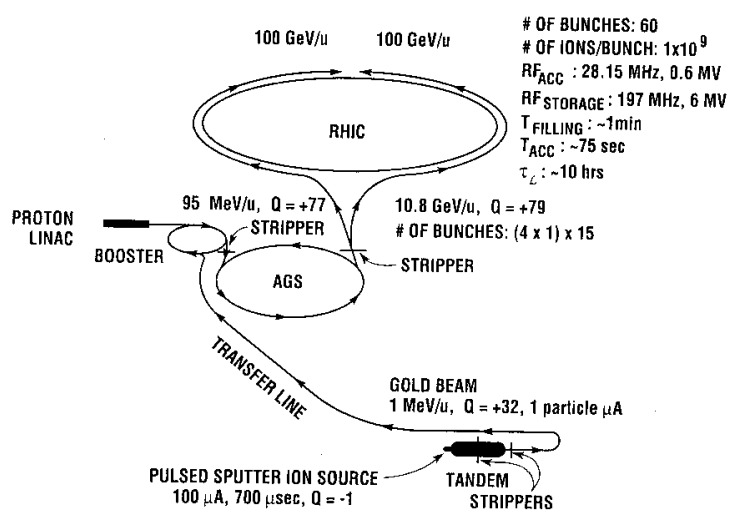

Figure 2: RHIC Acceleration Scenario for Au Beams.

RHIC lattice being at $\gamma_{\mathrm{T}}=24.7$, all ions, except protons, must go through this transition. The RHIC collider, indeed, is the first superconducting accelerator with slow ramp rate that involves the synchrotron phase transition. It is important to cross this transition rapidly in order to minimize the beam loss and the emittance growth. This can be accomplished either by rapid acceleration through it with resultant orbit jump to a larger radius or by a " $\gamma_{\mathrm{T}^{-}}$ jump", where sets of quadrupoles will be pulsed to change the tune of the machine, thus move the transition energy momentarily. For the year 2000 operation the former method was used for the lack of pulsed power supplies, while for the year 2001 run, the latter method will be used.

Four Siberian Snakes are installed in the RHIC rings (two in each ring, $180^{\circ}$ apart) as part of the BNL-RIKEN (Japan) collaboration. These Snakes [1] will make it possible to accelerate, store, and collide polarized proton beams, providing a unique opportunity to carry out the spin physics program at ultra-high center of mass energies.

The arrangement of detectors around the RHIC ring is shown in Fig. 3. There are two "major" detectors (STAR and PHENIX) and two "minor" detectors (PHOBOS and BRAHMS). Here, the qualifier "major" and "minor" refer to their scale or size, complexity, cost of construction, and size of the collaboration, and not to the depth of physics reach. These four detectors form a complementary set for the first round of experiments at RHIC.

The STAR detector, utilizes a solenoidal geometry with a large cylindrical Time-Projection Chamber (TPC) $(4 \mathrm{~m}$ in diameter and $4 \mathrm{~m}$ long), installed inside of a large solenoidal magnet, providing a close to $4 \pi$ solid angle tracking capability for charged particles from collisions. With the 3-dimensional tracking capability, i.e., projections on the end sectors giving the $x-y$ coordinates and drift time of ionization electrons giving the $\mathrm{z}$ coordinates of track segments, TPC can handle a large number of tracks from an event. The $\mathrm{dE} / \mathrm{dx}$ measurements of track segments allow an identification of particles over a significant momentum range of interest.
Barrel electromagnetic calorimeters are being added for photon detection.

The PHENIX detector consists of three magnetic spectrometers, i.e., a Central Spectrometer consisting of an axial field magnet and two detector arms, one on the west side and the other on the east side; and two Muon Arms, one on the north side and the other on the south side of the central spectrometer along the direction of beams. The basic concept of the Central Spectrometer is to cover selected solid angles with quasi-concentric layers of high-speed detectors of various types. The combination of these detectors will facilitate good tracking and the identification of leptons, hadrons, and photons. This concept will let PHENIX detect the phase transition in a number of observable signatures simultaneously. Only the Central Spectrometer was available for the 2000 physics runs.

The PHOBOS detector consists of a two-arm magnetic spectrometer (one arm for 2000 physics runs) as its central detector and a series of ring multiplicity detectors, which surround the beam pipe at various distances from the collision point and provide a close-to- $4 \pi$ solid angle coverage. The exclusive use of high resolution and high speed silicon micro-strip devices for the detection element

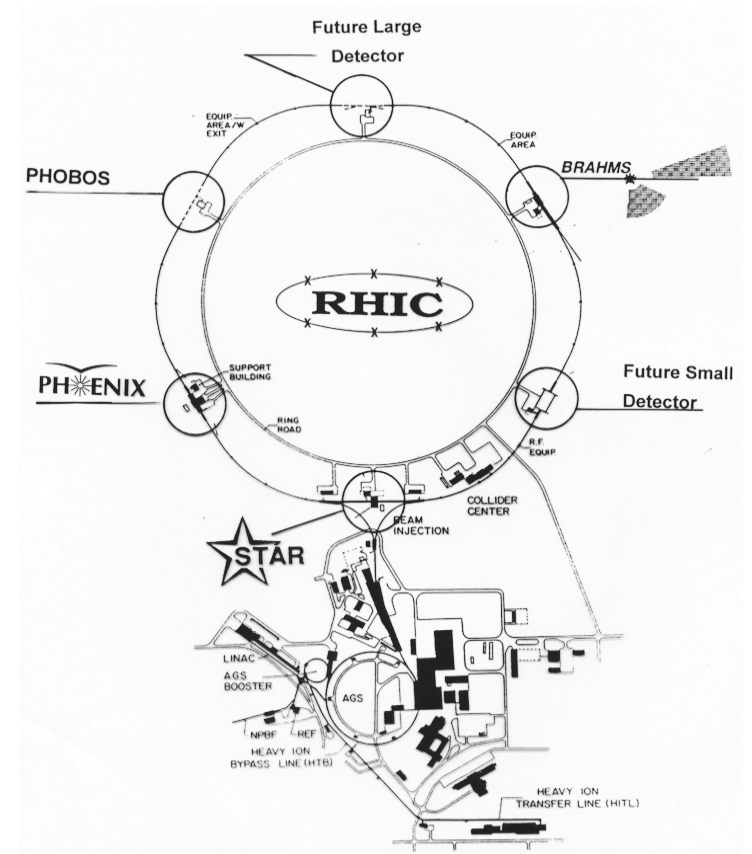

Figure 3: Arrangement of detectors along the RHIC ring.

will make the spectrometer "Table Top" size and also provide it a very high data rate capability for detection of charged hadrons and leptons in selected solid angles. The Time-of-Flight screens improve the particle identification capability of the detector.

The BRAHMS detector consists of a two-arm magnetic spectrometer, one in the forward direction for measurement of high momentum particles but with a small solid angle and the other on the side of the collision point for the mid-rapidity region. Both arms are movable to variable settings to cover wide ranges of kinematical 
regions. The technology used in this detector is more or less conventional in a sense that the design is quite similar to a spectrometer often used in a traditional fixed-target experiment at a high-energy accelerator facility. The spectrometers consist of room temperature narrow gap dipole magnets, drift chamber planes, other tracking devices, Cerenkov counters, and Time-of-Flight detectors.

\section{COMMISSIONING}

After meeting successive milestones such as the first sextant test in February 1997, the completion of magnet production in September 1998, and the assembly of the RHIC collider ring in January 1999, almost on schedule, the initial test to verify functionality of the collider system took place in June-September 1999. The actual commissioning with colliding beams, the commissioning of detectors, and the initial physics run took place during the late spring to summer of 2000.

For the operation in the year 2000, the cool-down of the collider ring began on March 10. After reaching the stable operating temperature of $4.6 \mathrm{~K}$, the Au beam was introduced into one of two rings (Blue Ring) on April 3 and to the other ring (Yellow Ring) on May 6. On May 20 the beam in the Blue Ring was accelerated through transition to approximately $60 \mathrm{GeV} / \mathrm{u}$. Acceleration and storage of beams in the Blue Ring took place on June 1, and that in the Yellow Ring on June 6. These led to the achievement of collisions in the STAR and PHOBOS

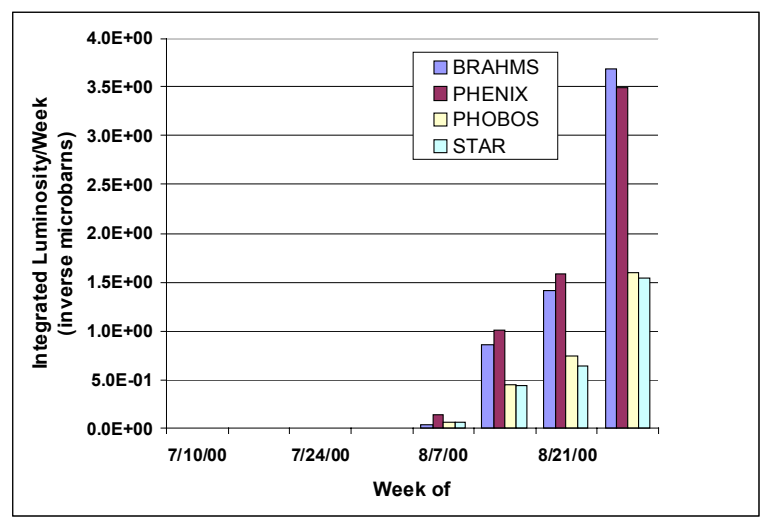

Figure 4: Integrated luminosity of $\mathrm{Au}-\mathrm{Au}$ collisions delivered per week.

detectors on June 12 at the beam energy of $28 \mathrm{GeV} / \mathrm{u}$ (or the total collision energy $56 \times \mathrm{A} \mathrm{GeV}$ ). Collisions in the PHENIX and BRAHMS detectors were observed 3 days later. Shortly thereafter, the beam energy was increased to $65 \mathrm{GeV} / \mathrm{u}$, realizing the center of mass collision energy of heavy ions some seven times higher than that achieved at the CERN SPS Pb beam operation. The target luminosity for the year 2000 run $(10 \%$ of the design luminosity) was reached at this energy on August 20, two weeks before the end of the heavy ion runs on September 5. The integrated luminosity per week, delivered to the four experiments, is shown in Figure 4. As can be seen in the figure, the integrated luminosity almost doubled each week and reached the total of $>\sim 6 \mathrm{mb}^{-1}$ to PHENIX and
BRAHMS, $>\sim 3 \mathrm{mb}^{-1}$ to STAR and PHOBOS, providing millions of relativistic $\mathrm{Au}-\mathrm{Au}$ collision events at the total collision energy of $130 \times \mathrm{A} \mathrm{GeV}$ (or $\sim 26.4 \mathrm{TeV}$ for $\mathrm{Au}-$ $\mathrm{Au})$.

As the collider was producing the collisions of $\mathrm{Au}$ beams, the four detectors were indeed ready to accept collision events and take data. Also ready were the largescale computer systems for data collection and analysis at the RHIC Computing Facility, and the physicists with computer software to tackle the analysis of complex events from heavy ion collisions.

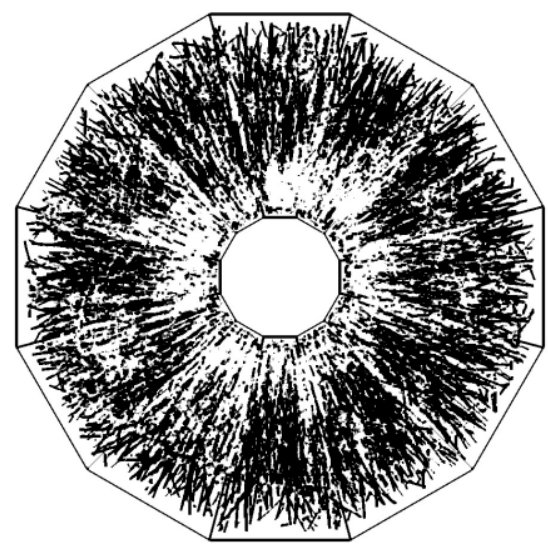

Figure 5: An Au-Au collision as detected by the STAR TPC (End view).

Fig. 5 shows by now well publicized STAR Au-Au events at center-of-mass energy of $130 \mathrm{xA} \mathrm{GeV}$ as recorded by its TPC. This picture demonstrates that this TPC, especially developed for high multiplicity events, can easily handle more than 1000 tracks, and measure the $\mathrm{dE} / \mathrm{dx}$ of individual tracks with a resolution of about $8 \%$. This capability combined with the effective mass reconstruction of unstable particles, based on the decay topology, allows STAR to identify and measure particles

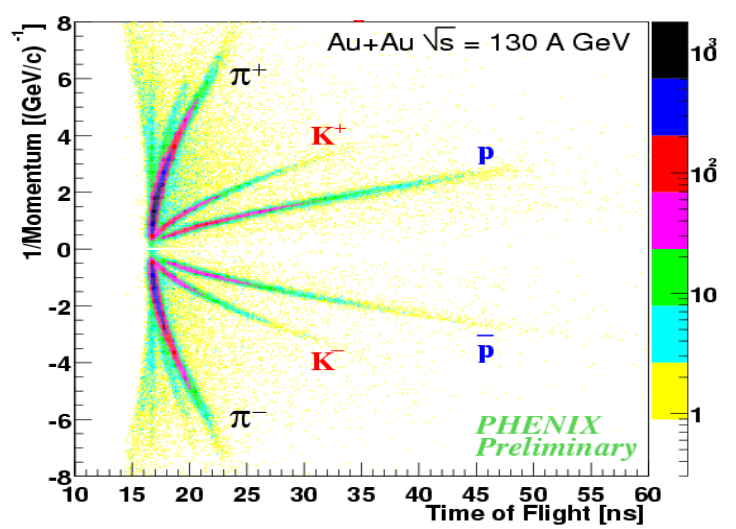

Figure 6: Particle Identification with Time-of-Flight with PHENIX Detector.

such as pion, kaon, proton, and their anti-particles as well as unstable particles such $\mathrm{K}^{*}, \varphi, \Lambda, \Xi$ etc.

Having multiple layers of various detectors in its central 
arms PHENIX can also do the track reconstruction and an identification of particles, as was the case with STAR. In addition, having the EM calorimeter and the ring imaging Cerenkov counter the PHENIX detector can identify and measure electrons and neutral pions. The result of timeof-flight measurements with the PHENIX detector is shown in Fig. 6.

\section{FIRST GLIMPSE OF NEW PHYSICS}

At the QM2001 conference that took place in January of this year, just 4 months after the 4 weeks of data taking that ended on September 5, 2000, RHIC research groups presented 31 physics papers on the first glimpse of the landscape at this exciting new frontier. [2] To date, 10 Phys. Rev. Lett. articles have been either published or submitted for publication. This speed by which significant physics results were produced from data obtained with the newly commissioned accelerator is unprecedented. What they have measured are global event characteristics such as particle multiplicity and its distribution, transverse energy measurement, and particle ratios. These measurements give valuable information on the energy density and temperature of the state produced by the collision. Other measurements included charged hadron spectra, neutral particle production, antiparticle/particle ratios, anisotropy of the particle distribution, and particle correlations, etc. A few examples of physics results are discussed below.

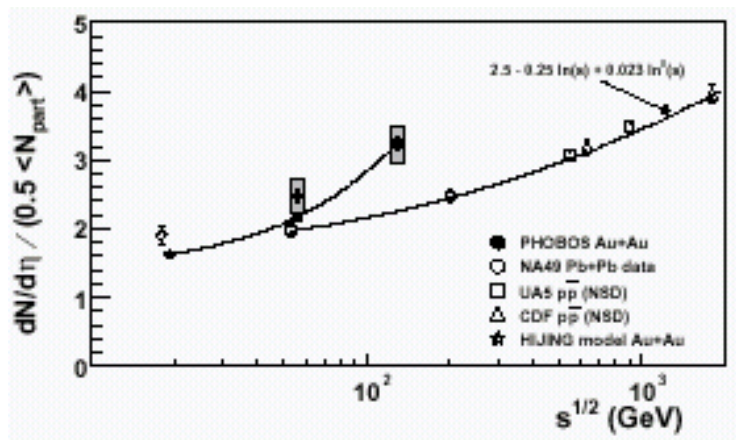

Fig 7: Data from the first physics publication from RHIC by PHOBOS Collaboration on particle multiplicity measurement.

Fig. 7 shows the charged particle multiplicity $(\mathrm{dN} / \mathrm{d} \eta)$ for $\mathrm{Au}-\mathrm{Au}$ collisions in the mid-rapidity region at the collision energies of $56 \mathrm{xA} \mathrm{GeV}$ and $130 \mathrm{xA} \mathrm{GeV}$, as measured by the PHOBOS detector. [3] In order to facilitate a comparison with other heavy ion experiments and $p-p$ data, the multiplicity values are normalized by the number of nucleon pairs participating in the collisions (a measure of centrality of collisions and equivalence of impact parameter). The values measured by PHENIX, STAR, and BRAHMS at 130xA GeV are consistent with PHOBOS measurement. There is a significant increase in the rate of charged particle production with energy within the RHIC energy region and a marked increase by a factor of approximately 1.5 from the CERN SPS Pb beam fixed target experiments. Another feature seen in this figure is that the multiplicity for $\mathrm{Au}-\mathrm{Au}$ is significantly higher than for $\mathrm{p}$-p collisions at the same collision energy.

PHENIX measured the multiplicity distribution and transverse energy distribution as a function of the number of participating nucleons. There is an excellent consistency between two distributions, which show that the number of particles produced grows faster than the number of participating nucleon pairs. This indicates a significant contribution of particle production from secondary and higher order interactions in the collision process. Using these analyses, PHENIX gave an estimate of the energy density for the Au-Au central collision at $130 \mathrm{xA} \mathrm{GeV}$ to be $4.6 \mathrm{GeV} / \mathrm{fm}^{3}$, about 1.6 times that at the CERN SPS $\mathrm{Pb}-\mathrm{Pb}$ experiments. [4]

With an assumption that a thermal equilibrium and chemical equilibrium have been reached in the central collisions of heavy ions, and given the freeze-out temperature of resulting fireballs and chemical (baryonic) potential, one can predict the ratios of various particles produced. The STAR collaboration carried out such an analysis, using $\mathrm{K}^{+} / \mathrm{K}^{-}$ratio that is $\sim 1$; p-bar $/ \mathrm{p}, \Lambda$-bar $/ \Lambda$, and $\Xi$-bar $/ \Xi$ ratios that range from $\sim 0.6$ to $\sim 0.8$; and $\mathrm{K}^{-} /\left(\mathrm{h}^{+}+\mathrm{h}^{-}\right), \mathrm{K}_{0} * / \mathrm{h}^{-}$, and $\mathrm{p}-\mathrm{bar} / \pi^{-}$ratios that are $\sim 0.1$. Their preliminary results showed that $\mathrm{Au}-\mathrm{Au}$ collisions at RHIC have reached the freeze-out temperature of $\mathrm{T}=$ $(2.2 \pm 0.2) \times 10^{12} \mathrm{~K}($ or $190 \mathrm{MeV})$ with baryonic potential $\mu_{\mathrm{B}} \sim 45 \mathrm{MeV}$.

The ratio of antiparticles to particles produced in heavy ion collisions is a good indicator of how baryon-rich (or baryon-free) the fireballs are. All four experiments measured the $p$-bar/p ratios. [5] Their results are very consistent, giving the ratio ranging from 0.55 to 0.62 with error less than \pm 0.05 . This value is significantly larger compared to $\sim 0.05$ at the CERN SPS energy (17A GeV) and suggests that we are approaching zero net baryon conditions at mid rapidity region.

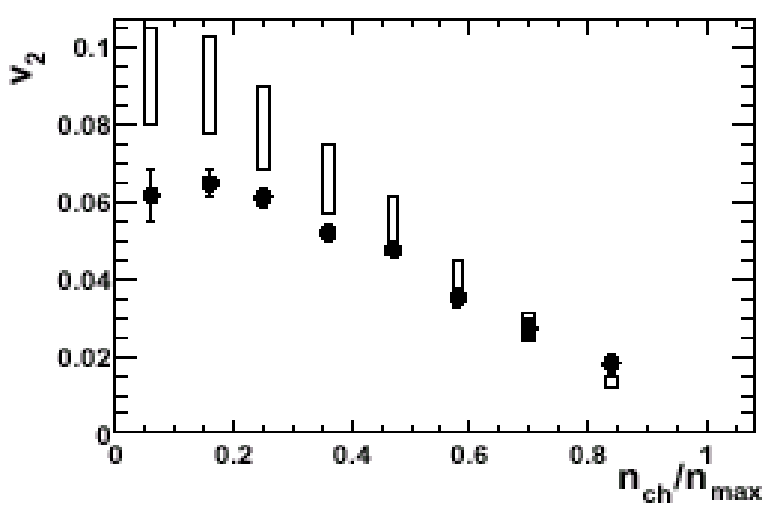

Figure 8: Elliptic flow (solid points) as a function of centrality defined as $n_{c h} / n_{\max }$. The open rectangles show a range of values expected for the value in the hydrodynamic limit.

Another observation of interest made by these experiments is anisotropy of particle distribution from the heavy ion collisions. Analyses of this anisotropy can give an indication on how the partons flow during the collision process. The analyses by STAR (Fig. 8) shows that there is a significant so called "elliptical flow" from 
$\mathrm{Au}-\mathrm{Au}$ collision at RHIC. [6] The result is more pronounced compared to the earlier analysis of the CERN SPS data. The measurement indicates that behavior of the state created by the relativistic heavy ion collisions at RHIC follows more closely the prediction of the hydrodynamic model.

\section{NEAR AND LONG TERM FUTURE}

As of this writing, both rings are at the operating cryogenic temperature and are poised to reach the $\mathrm{Au}$ beam energy of $100 \mathrm{GeV} / \mathrm{u}$. The run plan for FY 20012002 includes the $\mathrm{Au}-\mathrm{Au}$ collision physics programs at the collision energy of $200 \times \mathrm{A} \mathrm{GeV}$ during the months of July through September. October and November will be devoted to the commissioning and collisions of polarized proton beams at the collision energy of $200 \mathrm{GeV}$, before returning to the heavy ion program in December. The " $\gamma_{\mathrm{T}}$-jump" will be deployed for the transition crossing for the first time, and the $\beta^{*}$ at all collision points will be 10 $\mathrm{m}$ at injection and 2-10 $\mathrm{m}$ for collisions as required by each experiment. With the target luminosity at the design value, we hope to generate data 50 100 times those of FY 2000 run.

There are several paths for the luminosity upgrade in the future. A possibility of increasing the number of bunches per ring is already built into the collider as well as detectors. Another immediate upgrade path is to invoke the collision optics with $\beta^{*}=1 \mathrm{~m}$ at selected interaction points. This very tight focusing of beams is possible because of the very high field quality of the final focus quadrupoles at these locations.

The next stage of luminosity upgrade will be done with a higher bunch current. In this case, however, cooling of beams becomes necessary because the strong intra-beam scattering will result in rapid growth of emittance. An electron cooling technique using an energy recovery linac is being developed in collaboration with BINP, Novosibirsk.

\section{CONCLUSIONS}

After 17 years of gestation period, RHIC began to operate, opening a new frontier for nuclear research. The first glimpse of the landscape at this new frontier, as observed with collisions of $\mathrm{Au}$ ions at $2 / 3$ of the design collision energy, i.e., $130 \times \mathrm{A} \mathrm{GeV}$, already caught some tantalizing indications of unusual global behavior. These observations bode well for the exciting physics to come. Are we observing the onset of the transition to the QGP phase already at this energy? Unambiguous signatures of the phase transition rest in the understanding of rare processes. In this regard, with only $1 \%$ of integrated luminosity one would expect to collect during a year of operation, we are still very far from answering this question. I would like to take this opportunity to commend the members of the RHIC team, both BNL staff and collaborators, who have been involved in the design, construction, and commissioning of the RHIC collider and detector for their outstanding job. On behalf of the RHIC team, I express my gratitude to our funding agency, the US DOE, in particular the Nuclear Physics Division. The author also wishes to acknowledge with thanks the support the Project received from Brookhaven National Laboratory, both under the earlier management by Associate Universities Inc., and later by Brookhaven Science Associates. Last but not least, we thank other US and foreign funding agencies for their generous support and contributions to the detector projects and to the Spin physics capability at RHIC.

\section{REFERENCES}

[1] W. Mackay et al., "Commissioning and Future plans for Polarized Proton in RHIC", MOPA004 This Conference

[2] F. Videbaek, "BRAHMS Results from First RHIC Run”; W. A. Zajc, "PHENIX Overview"; G. Roland, "Results from the PHOBOS experiment at RHIC, J. Harris, "Overview of First Results from STAR, Proceedings of QM2001 Conference, to be published

[3] B. B. Back et al., "Charged particle multiplicity near mid-rapidity in central $\mathrm{Au}+\mathrm{Au}$ collisions at $\sqrt{\mathrm{S}}_{\mathrm{NN}}=$ 56 and $130 \mathrm{GeV}$ " submitted to Phys. Rev. Lett. 85, $3100(2000)$

[4] K. Adcox et al., "Centrality Dependence of Charged Particle Multiplicity in Au-Au Collisions at $\sqrt{\mathrm{S}}_{\mathrm{NN}}=$ 130 GeV" Phys. Rev. Lett. 86, 3500 (2001); K. Adcox et al., "Measurement of the mid-rapidity transverse energy distribution from $\sqrt{ }_{\mathrm{s}_{\mathrm{NN}}}=130 \mathrm{GeV}$ $\mathrm{Au}+\mathrm{Au}$ collisions at RHIC" to be published, Phys. Rev. Lett., May 2001.

[5] C. Adler et al., "Midrapidity Antiproton-to-Proton Ratio from $\mathrm{Au}+\mathrm{Au} \sqrt{\mathrm{S}}_{\mathrm{NN}}=130 \mathrm{GeV}$ " Phys. Rev. Lett. 86 pp. 4778-4782 (2001); I. G. Bearden et al., "Rapidity dependence of antiproton to proton ratios in $\mathrm{Au}+\mathrm{Au}$ collisions at $\sqrt{\mathrm{S}}_{\mathrm{NN}}=130$ GeV" submitted to Phys. Rev. Lett., April, 2001; B. B. Back et al., "Ratios of charged antiparticles to particles near mid-rapidity in $\mathrm{Au}+\mathrm{Au}$ collisions at $\sqrt{\mathrm{s}}_{\mathrm{s}}$ $\mathrm{NN}=130 \mathrm{GeV}$ " submitted to Phys. Rev. Lett., April, 2001.

[6] K. H. Ackermann et al., "Elliptic Flow in $\mathrm{Au}+\mathrm{Au}$ Collisions at $\sqrt{\mathrm{s}}_{\mathrm{NN}}=130 \mathrm{GeV}$ " Phys. Rev. Lett. 86 pp. 402-407 (2001). 